Cite this: J. Mater. Chem. C, 2013, 1, 3985

Received 8th March 2013

Accepted 26th April 2013

DOI: $10.1039 /$ c3tc30442f

www.rsc.org/MaterialsC
View Article Online

View Journal / View Issue

\title{
Photo-induced intramolecular charge transfer in an ambipolar field-effect transistor based on a $\pi$-conjugated donor-acceptor dyad $\dagger$
}

\author{
Raphael Pfattner, ${ }^{a}$ Egon Pavlica, ${ }^{\mathrm{b}}$ Michael Jaggi, ${ }^{\mathrm{c}}$ Shi-Xia Liu, ${ }^{\text {*c }}$ Silvio Decurtins, ${ }^{\mathrm{c}}$ \\ Gvido Bratina, ${ }^{b}$ Jaume Veciana, ${ }^{a}$ Marta Mas-Torrent ${ }^{* a}$ and Concepció Rovira ${ }^{a}$
}

A $\pi$-conjugated tetrathiafulvalene-fused perylenediimide (TTF-PDI) molecular dyad is successfully used as a solution-processed active material for light sensitive ambipolar field-effect transistors with balanced hole and electron mobilities. The photo-response of the TTF-PDI dyad resembles its absorption profile. Wavelength-dependent photoconductivity measurements reveal an important photo-response at an energy corresponding to a PDI-localized electronic $\pi-\pi^{*}$ transition and also a more moderate effect due to an intramolecular charge transfer from the HOMO localized on the TTF unit to the LUMO localized on the PDI moiety. This work clearly elucidates the interplay between intra- and intermolecular electronic processes in organic devices.

Donor-acceptor (D-A) dyads have been investigated for a long time in the field of molecular electronics since they can give rise to an intramolecular electron transfer promoted by an external stimulus like light irradiation. From a completely different perspective, these molecular systems have also been more recently the focus of attention in order to prepare ambipolar organic field-effect transistors (OFETs), which are of great interest in fabrication of organic integrated circuits. Ambipolar OFETs have been prepared by mixing two semiconductors ( $\mathrm{p}$ and $\mathrm{n}$ ), although unicomponent devices are more appealing for their ease of fabrication. ${ }^{\mathbf{1}}$ The covalent linkage of electron donor (D) and acceptor (A) moieties leads to a low HOMOLUMO gap which allows efficient injection of both holes and electrons. Very recently, high performance ambipolar OFETs based on soluble D-A polymers have been reported. ${ }^{2-4}$ However, ambipolar devices based on solution-processed D-A molecules are much more scarce and their performance lies well below their polymer counterparts. ${ }^{5,6}$ Furthermore, in all these molecular D-A based devices the influence of a possible intramolecular charge transfer (ICT) on the intermolecular transport has not been explored.

\footnotetext{
anstitut de Ciència de Materials de Barcelona (ICMAB-CSIC), Networking Research Center on Bioengineering, Biomaterials and Nanomedicine (CIBER-BBN), Campus UAB, 08193 Bellaterra, Spain. E-mail:mmas@icmab.es

${ }^{b}$ Laboratory of Organic Matter Physics, University of Nova Gorica, Vipavska 13, SI5000 Nova Gorica, Slovenia

${ }^{c}$ Departement für Chemie und Biochemie, Universität Bern, Freiestrasse 3, CH-3012 Bern, Switzerland. E-mail: shi-xia.liu@iac.unibe.ch

$\dagger$ Electronic supplementary information (ESI) available: Further information about experimental details, electrical device characterization, and photoconductivity measurements. See DOI: 10.1039/c3tc30442f
}

The fused tetrathiafulvalene-perylenediimide (TTF-PDI) D-A dyad with a largely extended $\pi$-conjugated skeleton shown in Fig. 1a has previously been described to exhibit a photoinduced ICT in solution. ${ }^{7}$ Here we report on the fabrication of OFETs based on this material and remarkably we demonstrate that such ICT phenomena can also be observed in the devices. A number of examples related to TTF derivatives as high performing active p-channel materials in OFETs have been reported. ${ }^{8-14}$ Similarly, PDI derivatives as active $\mathrm{n}$-channel materials have been known as well. ${ }^{15-17}$ In the present work, it is demonstrated that solution-processed films of TTF-PDI combine the transport characteristics of the separate units since they exhibit marked ambipolar behaviour with balanced hole and electron field-effect mobilities. Additionally, the photo-response of this device is carefully explored and high photoconductivity effects at specific light wavelengths are observed corresponding to characteristic electronic absorption bands of the PDI core and, very interestingly, to an ICT process from the TTF donor to the PDI acceptor units.

The HOMO-LUMO gap $\left(E_{\mathrm{g}}\right)$ of the TTF-PDI dyad was deduced from the onset of the lowest ICT absorption band measured in a thin-film on a quartz substrate and amounts to about $1.1 \mathrm{eV}$, which is in good agreement with the electrochemical HOMO-LUMO gap of $1.1 \mathrm{eV} .^{7}$ Such a small band-gap makes TTF-PDI a very promising ambipolar semiconductor for OFETs in which both holes and electrons are injected and transported.

OFETs based on solution-processed films of the TTF-PDI dyad were prepared by drop casting its $\mathrm{CH}_{2} \mathrm{Cl}_{2}$ solution $(c=$ $\left.1 \mathrm{mg} \mathrm{ml}^{-1}\right)$. The device configuration employed is a bottom-gate bottom-contact architecture (BGBC) using interdigitated gold 
(a)

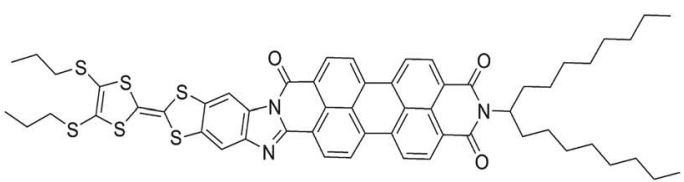

(b)

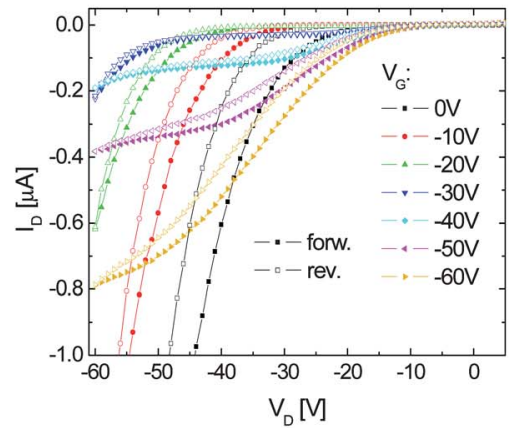

(c)

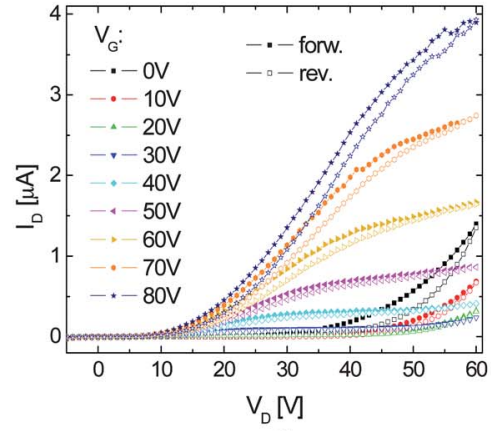

(d)

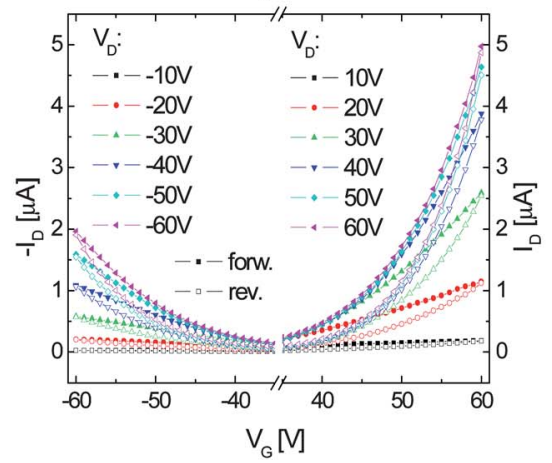

Fig. 1 (a) Chemical structure of the donor-acceptor dyad TTF-PDI, (b and c) output characteristics for the p-channel operation and n-channel operation, respectively, and (d) transfer characteristics in a linear plot for both $p$ and $n$ transport. Closed symbols represent forward voltage sweeps and open symbols represent reverse voltage sweeps.

source and drain electrodes. All the devices were prepared and analysed under inert conditions. In the output characteristics of the as-prepared devices, a distinct field-effect for the hole channel regime was observed (see ESI, Fig. S1†). In contrast, for the electron channel regime, no field-enhanced source-drain current was observed with increasing $V_{\mathrm{G}}$. When the devices were annealed in the dark under inert conditions, an increase of the device performance in the hole operation regime was observed. But much more interestingly, the devices started to show an enhanced source-drain current also in the electron channel regime when annealed at a temperature higher than $120^{\circ} \mathrm{C}$. In order to unravel the optimum annealing conditions for the fabrication of ambipolar OFETs with maximum balanced charge carrier mobility, the devices were annealed at different temperatures (see ESI, Fig. S2 and S3†). It turns out that the device displays maximum performance for ambipolar transport after annealing at $230{ }^{\circ} \mathrm{C}$ for 30 minutes. With increasing temperatures, particularly in the hole transport regime, there is a decrease of mobility caused by phase transition in the material (see ESI, Fig. S7†). Fig. 1(b) and (c) show the output characteristics measured for solution-prepared TTF-PDI OFETs after annealing at $230{ }^{\circ} \mathrm{C}$ for $30 \mathrm{~min}$. The corresponding transfer characteristics for this device are depicted in Fig. 1d. It should be highlighted that the devices were highly reproducible.

The maximum extracted field-effect mobility in the saturation regime at gate voltage $\left(V_{\mathrm{G}}\right)$ and source-drain voltage $\left(V_{\mathrm{D}}\right)$ $\left|V_{\mathrm{G}}\right|=\left|V_{\mathrm{D}}\right|=60 \mathrm{~V}$ was found to be $\mu_{\mathrm{h}+}=0.5 \times 10^{-3} \mathrm{~cm}^{2} \mathrm{~V}^{-1} \mathrm{~s}^{-1}$ and $\mu_{\mathrm{e}-}=1.5 \times 10^{-3} \mathrm{~cm}^{2} \mathrm{~V}^{-1} \mathrm{~s}^{-1}$ for holes and electrons, respectively. Similar threshold voltages were found for holes and electrons with $V_{\mathrm{th}}=-31 \mathrm{~V}$ and $V_{\mathrm{th}}=+32 \mathrm{~V}$, respectively. An "S"-shape in the current-voltage characteristics for both hole and electron channel regimes is observable at low $V_{\mathrm{D}}$ which might be related to charge injection barriers. However, the balanced hole and electron transport characteristics make TTFPDI highly appealing for the construction of ambipolar devices.

To demonstrate the feasibility of using solution-processed TTF-PDI ambipolar OFETs in complementary circuits, an inverter NOT gate was built. Fig. 2 shows the transfer characteristics of a complementary-like voltage inverter based on two ambipolar OFETs using TTF-PDI as an active material with the channel dimensions for T1 and T2: $W=10 \mathrm{~mm}$ and $L=10 \mu \mathrm{m}$. The insets show the calculated gain of up to 9 as well as a scheme of the inverter circuit. This result stays in fairly good agreement compared with inverter circuits prepared from other molecular ambipolar semiconductors. ${ }^{5}$

Photoconductivity was shown previously in OFETs with D-A dyads, ${ }^{18,19}$ PDI $^{20}$ as well as with TTF $^{21}$ as active materials. In addition to TTF-PDI solutions, TTF-PDI thin films reveal intense optical absorption bands over a wide spectral range.

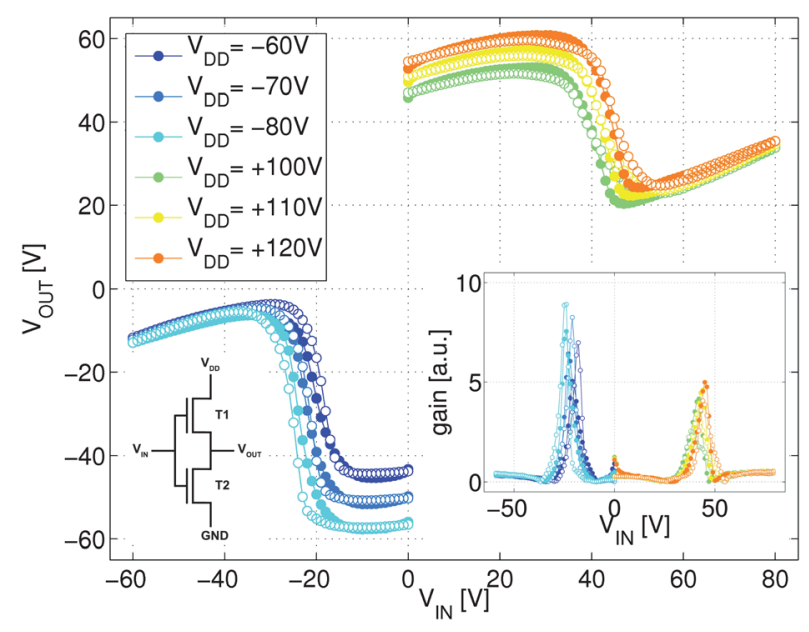

Fig. 2 Transfer characteristics of a complementary-like voltage inverter based on two ambipolar OFETs with TTF-PDI as an active material with the channel dimensions for T1 and T2: $W=10 \mathrm{~mm}$ and $L=10 \mu \mathrm{m}$. The insets show the calculated gain as well as the inverter circuit. Closed symbols represent forward voltage sweeps and open symbols represent reverse voltage sweeps. 
Consequently, the photoresponse of the prepared devices has been investigated. TTF-PDI-based OFETs were irradiated with light using a solar simulator with an intensity of $100 \mathrm{~mW} \mathrm{~cm}^{-2}$. A strong increase in source-drain current $\left(I_{\mathrm{D}}\right)$ up to almost $200 \%$ was observed, due to the photogeneration of charges. ${ }^{22}$ The maximum increase of the source-drain current $\left(I_{\mathrm{D}}\right)$ upon sample illumination takes place at gate voltage $V_{\mathrm{G}}=0 \mathrm{~V}$. This suggests that, as expected in this configuration, the number of photo-induced charge carriers in the experiment is much smaller compared to the gate-induced charge carriers at higher $\left|V_{\mathrm{G}}\right|$. Fig. 3a shows a typical measurement of $I_{\mathrm{D}}(t)$ normalized to the dark-current $I_{\mathrm{D} 0}=43 \mathrm{nA}$, obtained at $V_{\mathrm{D}}=+30 \mathrm{~V}$ and $V_{\mathrm{G}}=$ $0 \mathrm{~V}$. The source-gate leakage current $\left(I_{\mathrm{G}}\right)$ is also plotted in the graph, showing that it is not affected by the light irradiation. The response of the devices to light is reversible. These results demonstrate that, in addition to the gate voltage, incident light can be used to control the electric conductivity of TTF-PDI based devices. This photo-gating effect is highly desired for the emerging of multi-functional devices. ${ }^{23}$

In order to explore the wavelength dependent response of the $I_{\mathrm{D}}$, photoconductivity spectra of the TTF-PDI films were measured and compared with their UV-vis absorption spectra (Fig. 3b). The photoconductivity in terms of the external photonto-photogenerated charge conversion efficiency was measured as a function of incident photon energy. The external quantum efficiency (EQE) is evaluated as the ratio between the collected

(a)
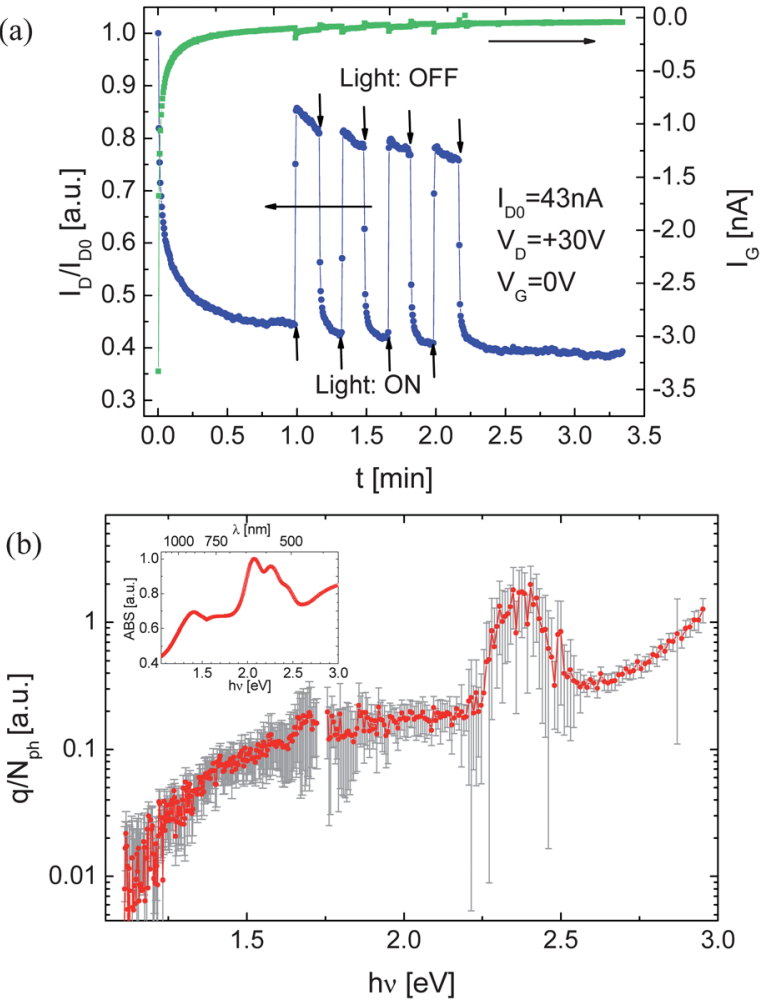

Fig. 3 (a) Electrical normalized response, $I_{D}(t) / /_{D O}$, of TTF-PDI OFET to light (light of solar simulator, intensity $=100 \mathrm{~mW} \mathrm{~cm}^{-2}$ ) exposure at $V_{\mathrm{G}}=0 \mathrm{~V}$ and $V_{\mathrm{D}}=+30 \mathrm{~V}$ with $I_{\mathrm{G}}$ leakage current. (b) External photon-to-photogenerated charge conversion efficiency as a function of incident photon energy and optical absorption spectrum measured for a TTF-PDI thin-film on a quartz slide (inset). photogenerated charge $(q)$ and a number of incident photons $\left(N_{\mathrm{ph}}\right)$. The $q / N_{\mathrm{ph}}$ ratio as a function of photon energy is shown in the logarithmic scale in Fig. 3b. The experiment was performed in two steps due to the employed laser and measurement setup: first in the range from $720 \mathrm{~nm}$ to $1100 \mathrm{~nm}$ and then between $420 \mathrm{~nm}$ and $710 \mathrm{~nm}$. Very importantly, the EQE spectrum highly agrees with the optical absorption spectrum measured for a TTF-PDI thin-film on a quartz slide (inset Fig. 3b).

The EQE in the region above $2.6 \mathrm{eV}(477 \mathrm{~nm})$ monotonically increases with photon energy due to the formation of free holeelectron pairs. A pronounced broad peak was found between $2.2 \mathrm{eV}$ and $2.5 \mathrm{eV}$ (564-496 $\mathrm{nm}$ ). In this region, EQE is almost one order of magnitude higher than it is in the remaining part of the spectrum. The EQE lineshape suggests that the broad peak represents a convolution of a number of narrow peaks. This spectral response range matches well with the intense absorption bands which are attributed to the PDI-localized electronic $\pi-\pi^{*}$ transitions. $^{7}$ Furthermore, an important increase in EQE between 1.1 and $1.5 \mathrm{eV}(1127-827 \mathrm{~nm})$ is in good agreement with the first absorption band of TTF-PDI, which is attributed to an intramolecular charge transfer (ICT) from the HOMO localized on the TTF donor to the LUMO localized on the PDI acceptor. ${ }^{7}$ To the best of our knowledge, this is the first time that the ICT in a D-A dyad in the solid state was visualized in this architecture by determining EQE as a function of photon energy. Essentially, we can state that the effect of an electronic intramolecular phenomenon on the intermolecular transport has been observed, which brings new perspectives into this field.

In summary TTF-PDI as a solution-processed active layer material in organic ambipolar OFETs shows high performance, and almost ideal ambipolar device characteristics when prepared under inert conditions. Balanced field-effect mobilities of up to $\mu_{\mathrm{h}+}=0.5 \times 10^{-3} \mathrm{~cm}^{2} \mathrm{~V}^{-1} \mathrm{~s}^{-1}$ and $\mu_{\mathrm{e}-}=1.5 \times$ $10^{-3} \mathrm{~cm}^{2} \mathrm{~V}^{-1} \mathrm{~s}^{-1}$ for holes and electrons, respectively, were found. Moreover, using these OFETs an inverter circuit was build indicative of possible applications of the TTF-PDI dyad for complementary organic circuits. Importantly, they are sensitive to light exposure with a completely reversible response upon illumination with the full solar spectrum. The EQE spectral response of the TTF-PDI dyad resembles its absorption profile. Wavelength-dependent photoconductivity measurements reveal a pronounced effect in the energy range from 2.2 to $2.5 \mathrm{eV}$ which corresponds to PDI-localized electronic $\pi-\pi^{*}$ transitions and, very importantly, an increase in the photoconductivity between 1.1 and $1.5 \mathrm{eV}$ which is attributed to an intramolecular charge-transfer from TTF to PDI moieties. This study has identified the high potential of the TTF-PDI dyad for electronic and optoelectronic applications, and elucidates that intramolecular and intermolecular electronic processes can interplay in an organic-based device.

\section{Acknowledgements}

The authors thank the EU Large Project ONE-P (FP7-NMP-2007212311), ERC StG 2012-306826 e-GAMES, the Networking Research Center on Bioengineering, Biomaterials and 
Nanomedicine (CIBER-BBN), the DGI (Spain) with project POMAS CTQ2010-19501/BQU and Generalitat de Catalunya 2009SGR516, as well as the Swiss National Science Foundation (grant no. 200020-130266/1). E.P. and G.B. acknowledge the support of the ESF Project GOSPEL (Ref. no: 09-EuroGRAPHENE-FP-001). G.B. acknowledges the Slovenian Research Agency program P1-0055. We are grateful to L. Martin-Samos Colomer for fruitful discussions.

\section{Notes and references}

1 J. Zaumseil and H. Sirringhaus, Chem. Rev., 2007, 107, 1296. 2 Z. Chen, M. Lee, R. Shahid Ashraf, Y. Gu, S. Albert-Seifried, M. Meedom Nielsen, B. Schroeder, T. Anthopoulos, M. Heeney, I. McCulloch and H. Sirringhaus, Adv. Mater., 2012, 24, 647.

3 A. J. Kronemeijer, E. Gili, M. Shahid, J. Rivnay, A. Salleo, M. Heeney and H. Sirringhaus, Adv. Mater., 2012, 24, 1558.

4 (a) J. Fan, J. D. Yuen, M. Wang, J. Seifter, J.-H. Seo, A. R. Mohebbi, D. Zakhidov, A. Heeger and F. Wudl, Adv. Mater., 2012, 24, 2186; (b) J. D. Yuen, J. Fan, J. Seifter, B. Lim, R. Hufschmid, A. J. Heeger and F. Wudl, J. Am. Chem. Soc., 2011, 133, 20799; (c) P. Sonar, S. P. Singh, Y. Li, M. S. Soh and A. Dodabalapur, Adv. Mater., 2010, 22, 5409.

5 P. H. Wöbkenberg, J. G. Labram, J.-M. Swiecicki, K. Parkhomenko, D. Sredojevic, J.-P. Gisselbrecht, D. M. de Leeuw, D. D. C. Bradley, J.-P. Djukic and T. D. Anthopoulos, J. Mater. Chem., 2010, 20, 3673.

6 (a) H. Krüger, S. Janietz, D. Sainova, D. Dobreva, N. Koch and A. Vollmer, Adv. Funct. Mater., 2007, 17, 3715; (b) R. P. Ortiz, H. Herrera, C. Seoane, J. L. Segura, A. Facchetti and T. J. Marks, Chem.-Eur. J., 2012, 18, 532; (c) S. Amriou, A. Mehta and M. R. Bryce, J. Mater. Chem., 2005, 15, 1232; (d) Q. Ye, J. Chang, K.-W. Huang and C. Chi, Org. Lett., 2011, 13, 5960; (e) F. Otón, R. Pfattner, E. Pavlica, Y. Olivier, G. Bratina, J. Cornil, J. Puigdollers, R. Alcubilla, X. Fontrodona, M. Mas-Torrent, J. Veciana and C. Rovira, CrystEngComm, 2011, 13, 6597.

7 M. Jaggi, C. Blum, N. Dupont, J. Grilj, S.-X. Liu, J. Hauser, A. Hauser and S. Decurtins, Org. Lett., 2009, 11, 3096.
8 M. Mas-Torrent and C. Rovira, Chem. Soc. Rev., 2008, 37, 827.

9 M. Mas-Torrent and C. Rovira, J. Mater. Chem., 2006, 16, 433.

10 M. Leufgen, O. Rost, C. Gould, G. Schmidt, J. Geurts, L. Molenkamp, N. Oxtoby, M. Mas-Torrent, N. Crivillers, J. Veciana and C. Rovira, Org. Electron., 2008, 9, 1101.

11 R. Pfattner, M. Mas-Torrent, I. Bilotti, A. Brillante, S. Milita, F. Liscio, F. Biscarini, T. Marszalek, J. Ulanski, A. Nosal, M. Gazicki-Lipman, M. Leufgen, G. Schmidt, L. W. Molenkamp, V. Laukhin, J. Veciana and C. Rovira, Adv. Mater., 2010, 22, 4198.

12 Naraso, J.-I. Nishida, S. Ando, J. Yamaguchi, K. Itaka, H. Koinuma, H. Tada, S. Tokito and Y. Yamashita, J. Am. Chem. Soc., 2005, 127, 10142.

13 Y. Takahashi, T. Hasegawa, S. Horiuchi, R. Kumai, Y. Tokura and G. Saito, Chem. Mater., 2007, 19, 6382.

14 X. K. Gao, Y. Wang, X. D. Yang, Y. Q. Liu, W. F. Qiu, W. P. Wu, H. J. Zhang, T. Qi, Y. Liu, K. Lu, C. Y. Du, Z. G. Shuai, G. Yu and D. B. Zhu, Adv. Mater., 2007, 19, 3037.

15 B. A. Jones, A. Facchetti, M. R. Wasielewski and T. J. Marks, J. Am. Chem. Soc., 2007, 129, 15259.

16 Y. Wen and Y. Liu, Adv. Mater., 2010, 22, 1331.

17 X. Zhan, A. Facchetti, S. Barlow, T. J. Marks, M. A. Ratner, M. R. Wasielewski and S. R. Marder, Adv. Mater., 2011, 23, 268.

18 M. Treier, A. Liscio, J. M. Mativetsky, M. Kastler, K. Müllen, V. Palermo and P. Samorì, Nanoscale, 2012, 4, 1677.

19 T. P. I. Saragi, M. Fetten and J. Salbeck, Appl. Phys. Lett., 2007, 90, 253506.

20 M. E. Gemayel, M. Treier, C. Musumeci, C. Li, K. Müllen and P. Samorì, J. Am. Chem. Soc., 2012, 134, 2429.

21 M. Mas-Torrent, P. Hadley, N. Crivillers, J. Veciana and C. Rovira, ChemPhysChem, 2006, 7, 86.

22 Bias stressing effects were found to be independent of illumination, which argues that the photoresponse is not due to charge de-trapping, but due to photogeneration of free charges.

23 J. Sayago, F. Rosei and C. Santato, Nat. Photonics, 2012, 6, 639. 